\title{
Research Article \\ Effects of Gelam Honey (Melaleuca cajuputi) on Alveolar Bone Loss in Experimental Periodontitis
}

\author{
N. Hamzah, ${ }^{1}$ S. A. Aziz, ${ }^{1}$ A. R. Fauzi, ${ }^{2}$ Y. A. Mohd Yusof, ${ }^{3}$ M. Razali, ${ }^{1}$ \\ N. Ibrahim, ${ }^{4}$ and B. Baharin ${ }^{1}$ \\ ${ }^{1}$ Department of Periodontology, Faculty of Dentistry, University Kebangsaan Malaysia, Jalan Raja Muda Abdul Aziz, \\ 50300 Kuala Lumpur, Malaysia \\ ${ }^{2}$ Department of Oral Biology, Faculty of Dentistry, University Kebangsaan Malaysia, Jalan Raja Muda Abdul Aziz, \\ 50300 Kuala Lumpur, Malaysia \\ ${ }^{3}$ Department of Biochemistry, Faculty of Medicine, University Kebangsaan Malaysia, Jalan Raja Muda Abdul Aziz, \\ 50300 Kuala Lumpur, Malaysia \\ ${ }^{4}$ Department of Oral Medicine and Oral Pathology, Faculty of Dentistry, Universiti Kebangsaan Malaysia, \\ Jalan Raja Muda Abdul Aziz, 50300 Kuala Lumpur, Malaysia
}

Correspondence should be addressed to N. Hamzah; drshikinhamzah@yahoo.com

Received 25 April 2014; Revised 10 August 2014; Accepted 25 September 2014; Published 10 November 2014

Academic Editor: Adrian Kasaj

Copyright (C) 2014 N. Hamzah et al. This is an open access article distributed under the Creative Commons Attribution License, which permits unrestricted use, distribution, and reproduction in any medium, provided the original work is properly cited.

Gelam honey has been shown to exhibit antioxidant and anti-inflammatory activities in animal model. The aim of this study was to determine the effects of Gelam honey (Melaleuca cajuputi) on alveolar bone level in experimental periodontitis. Thirty male Sprague-Dawley rats were used in this study and randomly divided into four groups: ligated saline (LS), ligated honey (LH), nonligated saline (NLS), and nonligated honey (NLH). Fifteen days after supplementation with Gelam honey $(3 \mathrm{~g} / \mathrm{kg})$, the rats were sacrificed and alveolar bone level was determined by radiography and histomorphometry. The number of osteoclasts was also calculated for all groups. Both radiographic and histomorphometric analyses showed that alveolar bone resorption was severely induced around the ligated molar in the LS and LH groups. There was no significant difference in alveolar bone level between the LS and LH groups. However, there was a nonsignificant reduction of osteoclast number by $15.2 \%$ in LH group compared to LS group. In the NLH group, there was less alveolar bone resorption and the number of osteoclasts was reduced by $13.2 \%$ compared to NLS group. In conclusion, systemically supplemented Gelam honey was shown to have the potential of reducing osteoclast activity in the experimental periodontitis rats, even though the effect on alveolar bone level was not well demonstrated and it warrants further research.

\section{Introduction}

Periodontitis is one of the most common chronic inflammatory diseases among adults worldwide. It is characterized by gingival inflammation, periodontal pocket formation, and bacterial plaque formation which lead to alveolar bone destruction and tooth loss. The pathogenesis of periodontitis involves the presence of plaque that may initiate local inflammatory reaction in a predisposed host, thus evoking edema, cell influx, and release of inflammatory mediators which ultimately leads to alveolar bone loss [1]. Among the inflammatory mediators, PGE2, Il-1, and TNF- $\alpha$ seem to be the important mediators as causative agents of tissue destruction [2]. In periodontal disease, the response of bone to local factors, produced by the inflammatory process, changes the bone remodeling balance, with a net effect of alveolar bone resorption and loss of attachment [3].

Alveolar bone resorption can be measured using various methods, that is, radiograph [4], two-dimensional/threedimensional imaging $[5,6]$, morphometric method [6], histomorphometric method, [7] and immunocytochemistry [8]. Radiographically, alveolar bone loss can be determined by the radiograph density changes usually seen at the interproximal area. The changes are measured from the cementoenamel 
junction (CEJ) to the alveolar bone crest (ABC). Radiograph helps to determine the severity of periodontitis and bonerelated damage but with limited sensitivity. The histological analysis is considered as the gold standard, accurate, and widely accepted technique in measuring alveolar bone loss in vitro $[9,10]$. Histomorphometrically, bone loss was measured from the cementoenamel junction (CEJ) to the alveolar bone crest $(\mathrm{ABC})$, while, for osteoclast activity, it is determined by morphologically quantifying the number of osteoclasts $[7,11]$.

Conventional treatment modalities for periodontitis include mechanical removal of microbial plaque and calculus by scaling and root debridement and in some patients may need adjunctive chemotherapeutics [12]. Chemotherapeutics may come in form of drugs or certain agents with inhibitory properties either antibacterial, antiseptic, antimicrobial, anti-inflammation, or antioxidant. The inhibitory agents involve natural products such as vitamin C, vitamin $\mathrm{D}$, calcium, and also sources of polyphenolic, flavonoids, and carotenoids from natural fruit/vegetable/berry intake [13]. Several reviews [14, 15] have concluded that antimicrobials used as adjunct to mechanical therapy improve therapeutic outcome. Chlorhexidine mouthwash is one of the most effective antimicrobial agents for plaque control [16], hence, to prevent periodontal disease. Recent interest in natural and alternative medicine has prompted the use of herbal and natural products in dentistry field. Nayak et al. [17] reported mouthwash containing Manuka honey had antimicrobial effect as significantly comparable to chlorhexidine mouthwash. Aloe vera gel is another example of natural product used as a local delivery medicament in treating periodontitis [18].

Honey is considered as the oldest medicinal food and has continued to be used throughout the ages. Gelam honey is a Malaysian local honey taken from Gelam tree, Melaleuca cajuputi. It has high phenolics and flavonoids contents such as ellagic acid, chlorogenic acid, ferulic acid, quercetin, and chrysin [19]. Gelam honey has also been shown to have antibacterial, anti-inflammatory, antioxidant, and healing properties [20-23]. However, to the best of our knowledge, the therapeutic use of Gelam honey in dentistry field, specifically for treating periodontal disease, has never been explored. It is envisaged that Gelam honey, with its high phenolics and flavonoids content, will alleviate the inflammatory mediators raised during periodontal disease, thus preventing bone destruction in an established lesion.

\section{Material and Methods}

2.1. Experimental Procedures. All procedures involving animals were approved by the animal ethics committee of University Kebangsaan Malaysia, (FD/PERIOD/2011/BADIAH/ 23-NOVEMBER/395-NOVEMBER-2011-JULY-2012). We used 30 male Sprague-Dawley rats weighing from 250 to $350 \mathrm{~g}$. Rats were used because the periodontal anatomy of the molar regions is similar to human and they are easier to handle compared to other animal models such as dogs or nonhuman primates. The rats were divided into four groups $(N=7)$ : ligated rats fed with saline (LS), ligated rats fed with Gelam honey (LH), nonligated rats fed with saline (NLS), and nonligated rats fed with Gelam honey (NLH). Gelam honey given to the LH and NLH groups was diluted according to the body weight. The dose needed was $3 \mathrm{~g} / \mathrm{kg}$ daily. Meanwhile for saline, the amount given to rats in LS and NLS groups was $3 \mathrm{~g} / \mathrm{kg}$ daily. Gelam honey and saline were fed to the rats via oral gavage using stainless steel feeding needle from day 1 to day 15 once daily every morning. For baseline purpose, we sacrificed two rats at 0 day. The periodontal disease was induced by placing silk ligature around the left mandibular first molar. Ligature method has been accepted as useful experimental model in assessing alveolar bone loss in periodontitis as described by Toker et al. [7], Liberman et al. [24], and Guimarães et al. [25]. All rats were sacrificed at day 15 by cardiac puncture followed by cervical dislocation. Mandible was surgically dissected and immediately fixed in 10\% paraformaldehyde.

2.2. Radiograph Measurement of Alveolar Bone Level. Hemimandible was placed on a radiographic box at a distance of $20 \mathrm{~cm}$ from the X-ray source. Digital radiographic analysis of control and test groups was performed with X-ray machine of a $40 \mathrm{~kW}$ exposure for $0.01 \mathrm{sec}$ and image analysis software used was Trophy Windows Imaging software. For radiograph image, alveolar bone loss was measured as the distance between cementoenamel junction (CEJ) and the alveolar bone crest (ABC). Three measurements were taken at mesial, distal, and one furcation area and presented as distances in millimetre $(\mathrm{mm})$.

2.3. Tissue Preparation for $H \& E$ Staining. The mandibles with intact surrounding tissue from each rat were dissected and fixed in freshly prepared $10 \%$ paraformaldehyde. After fixation, the specimens were decalcified in $10 \%$ formic acid for a minimum of three weeks, as described by Toker et al. [7]. The $10 \%$ formic acid was changed daily. After decalcification, the tissue was processed by an automated tissue processor. The tissue block then embedded in paraffin for cryostat sectioning. The paraffin block was then cut into mesiodistal sections $(5 \mu \mathrm{m})$ parallel to the long axis of the first molar mandibular teeth. The above preparation procedure was described by Li and Amar [6]. Hematoxylin and eosin (H\&E) staining was done for the tissues sections.

2.4. Histomorphometric Measurement of Alveolar Bone Level and Osteoclast Activity. For the histomorphometric image, measurement of bone loss was made from cementoenamel junction (CEJ) to the alveolar bone crest $(\mathrm{ABC})$ in the interproximal area between first and second mandibular molar, at two different points, that is, distal of first molar and mesial of second molar. For osteoclast activity, numbers of osteoclast cells were manually counted within analysis area of $10 \mu \mathrm{m} \times 10 \mu \mathrm{m}$ from alveolar bone crest (ABC) downward at the interproximal area between first and second mandibular molar.

2.5. Reproducibility. Both analysis methods were carried out by one examiner and intraexaminer reproducibility was 


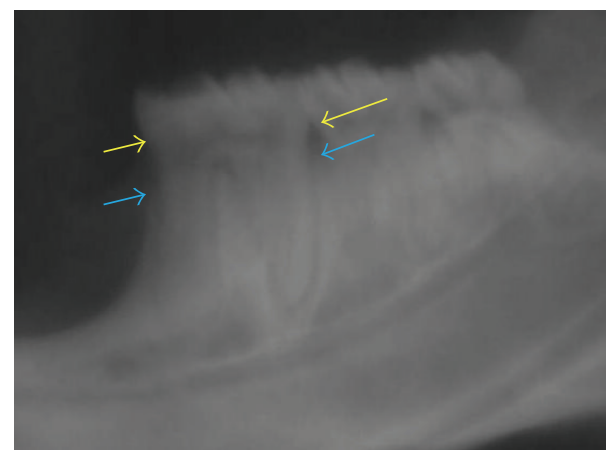

FIGURE 1: Radiograph images of the left mandible. Cementoenamel junction represented with yellow arrow and alveolar bone crest represented with blue arrow. Image showing normal structure of alveolar bone in rats at 0 day.

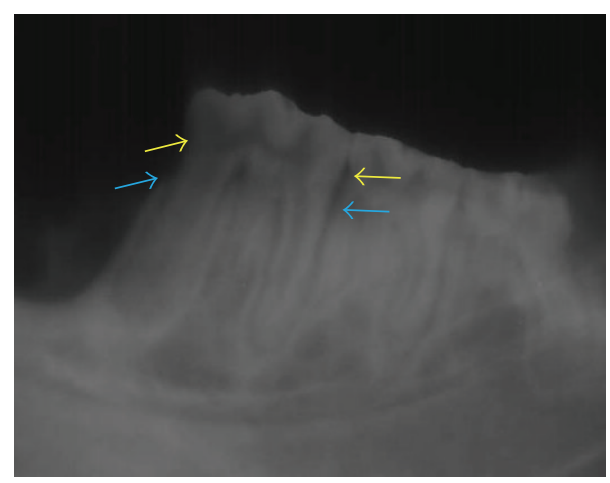

FIGURE 2: Radiograph images of the left mandible. Cementoenamel junction represented with yellow arrow and alveolar bone crest represented with blue arrow. Image showing normal structure of alveolar bone in rats fed with saline at 15 days.

carried out within one-week interval. Intraclass correlation coefficient statistic was used to check for reproducibility and revealed a very high correlation $($ ICC $=0.98)$.

2.6. Statistical Analysis. Data was analysed using IBM SPSS data editor version 20.0. All values were expressed as mean \pm standard error mean (SEM) of $n$ observation where $n$ represent the number of animals studied. One-way analysis of variance (ANOVA) between groups was used to compare alveolar bone loss measurement, followed by Tukey's posthoc test to check for significance between groups. $P$ value $<$ 0.05 was considered as statistically significant in this study.

\section{Results}

3.1. Radiographic Analysis. Figures 1 and 2 show normal structure of alveolar bone in rats fed with normal saline at 0 and 15 days, while Figure 3 shows normal structure of alveolar bone in rats fed with Gelam honey.

The presence of the silk ligature around the first left mandibular molar induced an inflammatory reaction to the periodontal tissue causing alveolar bone destruction which was clearly observed after 15 days of ligation (Figure 4).

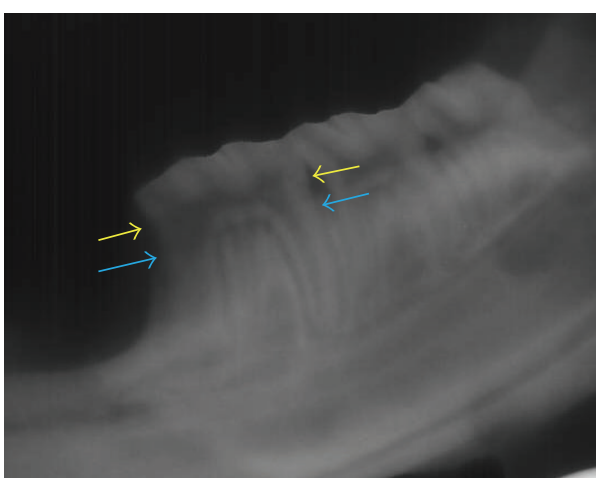

FIGURE 3: Radiograph images of the left mandible. Cementoenamel junction represented with yellow arrow and alveolar bone crest represented with blue arrow. Image showing normal structure of alveolar bone in rats fed with Gelam honey at 15 days.

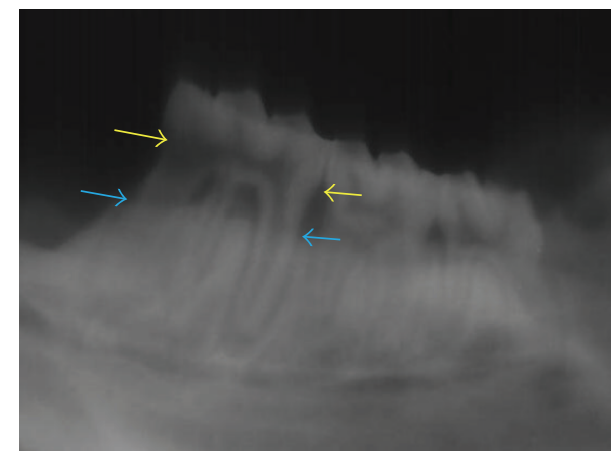

FIGURE 4: Radiograph images of the left mandible. Cementoenamel junction represented with yellow arrow and alveolar bone crest represented with blue arrow. Image showing alveolar bone destruction in rats fed with saline at 15 days.

The supplementation of Gelam honey did not show any modulating effect to the damaged alveolar bone (Figure 5).

Analysis of radiographs measurement of alveolar bone level revealed increased alveolar bone resorption in ligated groups (LS) and ligated group supplemented with honey (LH) compared to the baseline (0 day) and healthy/nonligated groups (NLS and NLH) as shown in Figure 6.

\subsection{Histomorphometric Analysis}

3.2.1. Alveolar Bone Level. H\&E-stained sections were evaluated for changes in alveolar bone level (Figures 7(a) to $7(\mathrm{e})$ ). Figures 7 (a) to 7(c) show normal alveolar bone level in control groups (baseline rats and nonligated rats fed with saline and Gelam honey $3 \mathrm{~g} / \mathrm{kg}$ at 15 day). Figures $7(\mathrm{~d})$ and 7 (e) show marked increase of alveolar bone resorption in ligated rats fed with saline and ligated rats fed with Gelam honey $3 \mathrm{~g} / \mathrm{kg}$ at 15 day. Supplementation of Gelam honey did not show inhibition effect on the alveolar bone destruction in periodontitis rats. Similar to the radiograph findings, the histomorphometric analysis (Figure 8) shows increased alveolar bone resorption in ligated groups (LS and LH) compared to the baseline ( 0 day) and nonligated groups (NLS and NLH). 


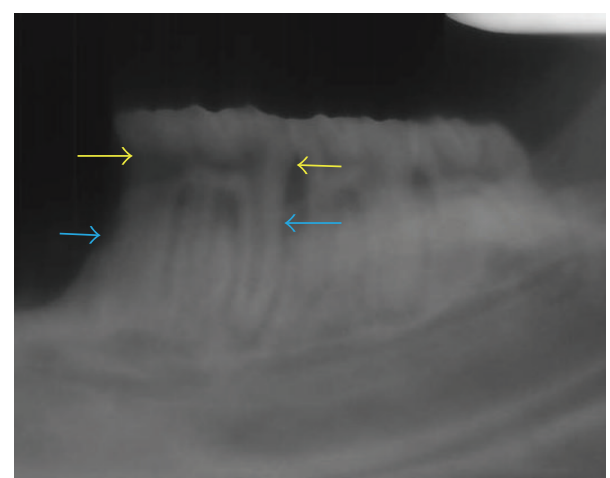

FIGURE 5: Radiograph images of the left mandible. Cementoenamel junction represented with yellow arrow and alveolar bone crest represented with blue arrow. Image showing alveolar bone destruction in rats fed with Gelam honey at 15 days.

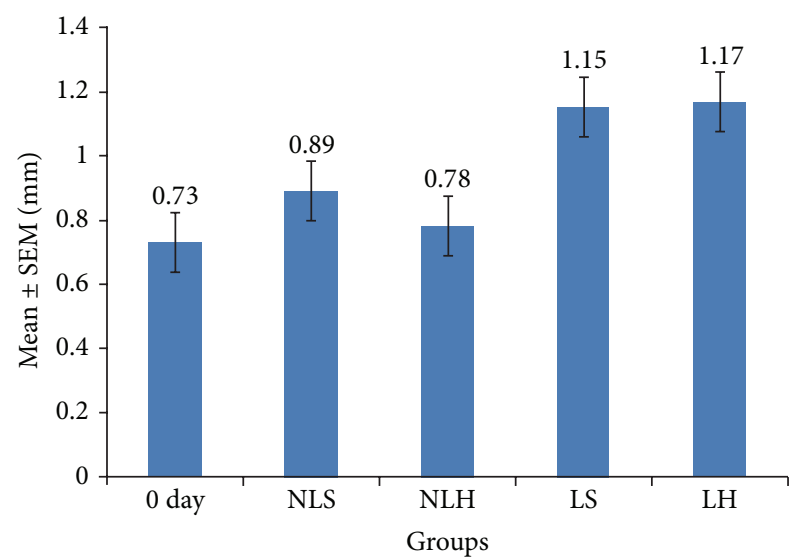

FIGURE 6: Mean \pm SEM $(\mathrm{mm})$ for radiographic measurement of alveolar bone level among groups. NLS: nonligated saline, NLH: nonligated honey, LS: ligated saline, and LH: ligated honey.

3.2.2. Osteoclast Activity. Osteoclasts $(n=5)$ were detected in the baseline rats and increased numbers of osteoclasts (NLS = $7.57, \mathrm{NLH}=5.47, \mathrm{LH}=7.4$, and the highest in $\mathrm{LS}=11.29$ ) were detected in all the other groups. The ligated groups (LS and $\mathrm{LH}$ ) showed more numbers of osteoclasts compared to the nonligated groups (NLS and NLH).

The H\&E staining slides were also evaluated for osteoclast activity in each group (Figures 9(a)-9(e)). Figure 9(a) shows no osteoclast activity in the baseline rat at zero day. Figures 9(b) and 9(c) show few osteoclasts in nonligated saline (NLS) and nonligated honey (NLH) at 15 days. The number of osteoclasts are less in the NLH group compared to NLS, while Figures 9(d) and 9(e) show increased osteoclast activity in the ligated rats fed with saline and ligated rats fed with Gelam honey $3 \mathrm{~g} / \mathrm{kg}$ at 15 day. The supplementation of Gelam honey was able to reduce osteoclast activity and zone of bone destruction compared to LS group.

Figure 10 showed the number of osteoclast from all the groups. Osteoclasts were detected in small numbers in the baseline rats (5) and higher numbers of osteoclasts were detected in NLS (7.57), NLH (5.47), and LH group (7.4), and the highest numbers were detected in LS group (11.29).

The reduction number of osteoclasts was observed between the ligated and nonligated groups supplemented with honey. Gelam honey shows $15.2 \%$ reduction of osteoclasts compared to ligated rats fed with saline, while $13.2 \%$ reduction was found in the nonligated groups. However, these findings were found not statistically significant $(P=$ 0.651 and $P=0.475$, resp.) as shown in Figure 11 .

\section{Discussion and Conclusion}

In periodontal disease, the response of bone to local factors, that is, bacterial plaque and calculus, produced by the inflammatory process, changes the bone remodeling balance, with a net effect of bone resorption and loss of attachment [26]. In this study, we have been able to show that the placement of ligature around the first mandibular molar induced marked bone loss in the ligated-groups rats. Our data are in accordance with previous studies by Samejima et al. [27], de Lima et al. [11], and Guimarães et al. [25] that also showed marked bone loss in ligature-induced periodontitis rats after 15 days. According to a study by Kuhr et al. [10] ligature-induced bone loss is particularly visible on day 15 whereby a $200-300 \mu \mathrm{m}$ increase in the cementoenamel junction $(\mathrm{CEJ})$ to alveolar bone crest $(\mathrm{ABC})$ distance in ligature effect zone is observed.

Our study shows that Gelam honey was able to decrease the number of osteoclast both in the diseased and in the healthy groups. Similar findings were reported by Toker et al. [7] and Cai et al. [28] where the supplementation of propolis and baicalin also reduced the number of osteoclasts in periodontitis induced rats.

According to Kassim et al. [23], Gelam honey and its extracts, honey methanolic extract (HME) and honey ethyl acetate extract (HEAE), contain high concentrations of phenolic compounds, especially ellagic acid and gallic acid which have been reported to inhibit iNOS (inducible nitric oxide synthase) and COX2 (cyclooxygenese2), decrease histamine release, and suppress proinflammatory cytokine production in macrophage and P-selectin-mediated inflammation both in vitro and in vivo $[29,30]$. $\mathrm{NO}$ (nitric oxide) is known to be an important mediator of acute and chronic inflammation. The iNOS is upregulated in response to inflammatory and proinflammatory mediators, and their products can influence many aspects of the inflammatory cascade [23]. We postulate that the inflammation cascade was altered by giving Gelam honey to the diseased and healthy groups. All the proinflammatory cytokines were reduced thus leading to reduction of osteoclasts activation or osteoclasts maturation.

Besides that, we also observed that the destruction of alveolar bone loss seen was consistent with the increased number of osteoclasts in the diseased groups. The same trend in osteoclastic activity was reported by other studies $[6,8,28]$, where the increase in osteoclast number and activity correlated well with the amount of alveolar bone resorption. $\mathrm{Li}$ and Amar [6] discussed in their study that periodontal tissue destruction increased with obvious inflammatory infiltration 


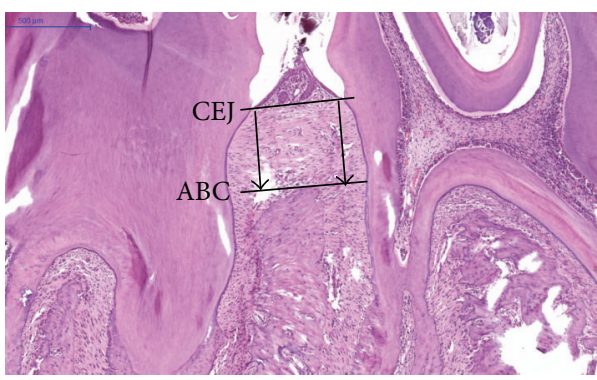

(a)

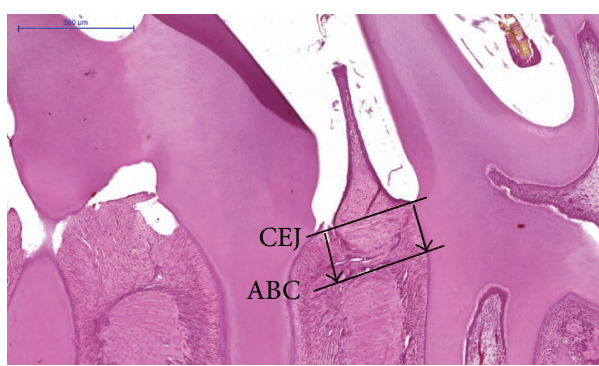

(c)

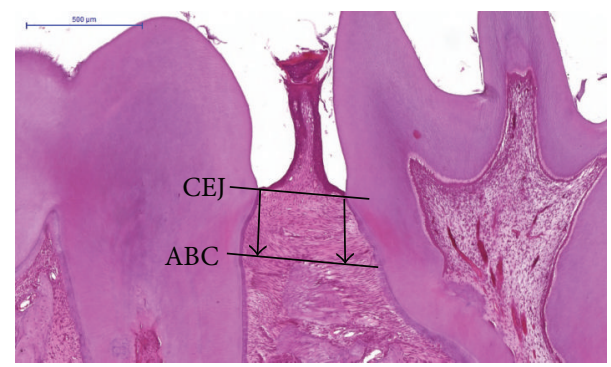

(b)

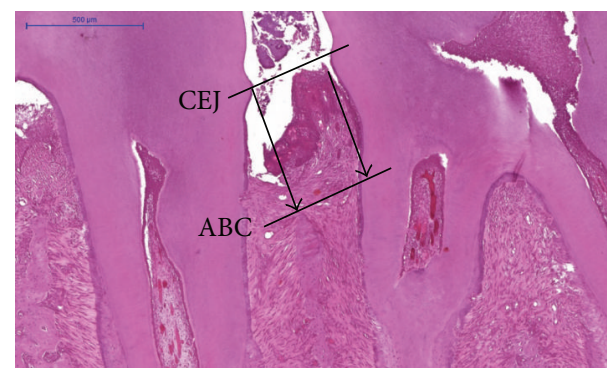

(d)

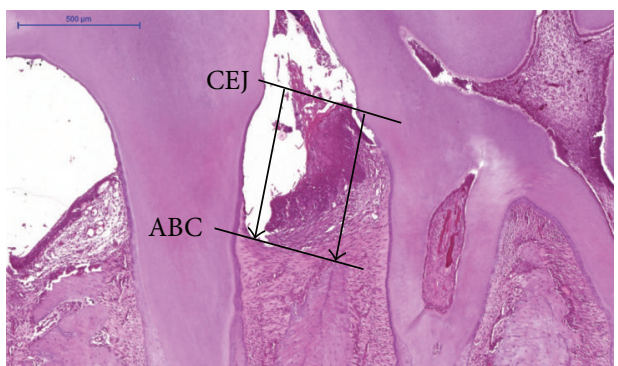

(e)

FIGURE 7: H\&E of alveolar bone of baseline, healthy, and diseased rats (at 5x magnification). CEJ: cementoenamel junction; ABC: alveolar bone crest. (a) shows normal alveolar bone level in control groups of baseline rats. (b) shows normal alveolar bone level in control groups of nonligated rats fed with saline $3 \mathrm{~g} / \mathrm{kg}$ at 15 days. (c) shows normal alveolar bone level in control groups of nonligated rats fed with Gelam honey $3 \mathrm{~g} / \mathrm{kg}$ at 15 days. (d) shows marked increase of alveolar bone resorption in ligated rats fed with saline $3 \mathrm{~g} / \mathrm{kg}$ at $15 \mathrm{days}$. (e) shows marked increase of alveolar bone resorption in ligated rats fed with Gelam honey $3 \mathrm{~g} / \mathrm{kg}$ at 15 days.

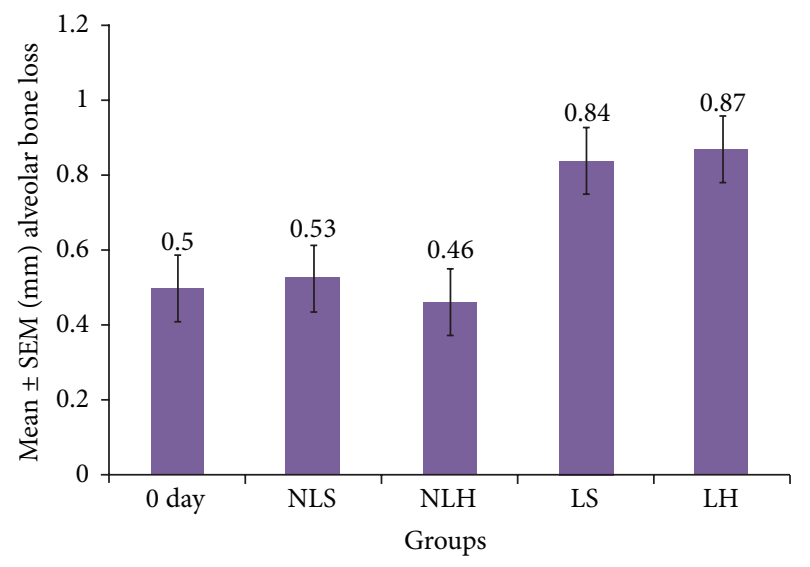

FIGURE 8: Mean \pm SEM (mm) for histomorphometric measurement of alveolar bone level among groups. NLS: nonligated saline, NLH: nonligated honey, LS: ligated saline, and LH: ligated honey. and the number of osteoclasts around the alveolar bone surface also increased after infection.

Analysis of alveolar bone loss measurement in the modulated rats was similar radiographically and histomorphometrically, where the diseased groups showed marked alveolar bone resorption compared to the healthy group and this is in accordance with Li and Amar [6]. Both analyses showed no reduction of alveolar bone loss with supplementation of Gelam honey within the diseased group, but some reduction was shown in the healthy group. From our study, bone loss in the diseased group was clearly increased than in the healthy group. However, we were unable to demonstrate reduction of alveolar bone resorption with Gelam honey supplementation. This may suggest that microscopic changes are seen first before macroscopic changes. Furthermore, our results are similar to the study by Guimarães et al. [25] on the effects of curcumin on modulated periodontitis. They reported that, after 15 days of supplementation, curcumin did not prevent 


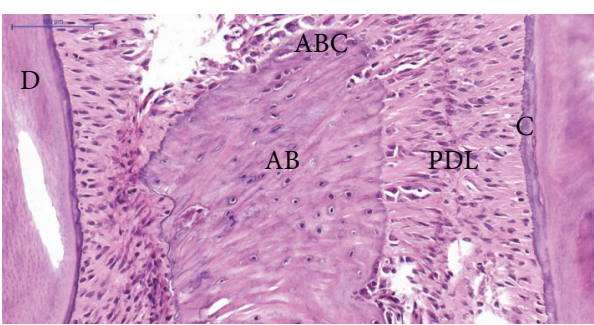

(a)

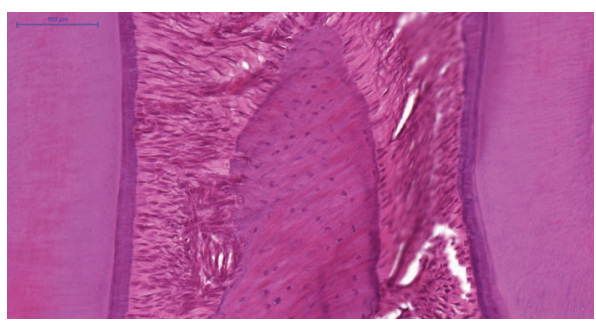

(c)

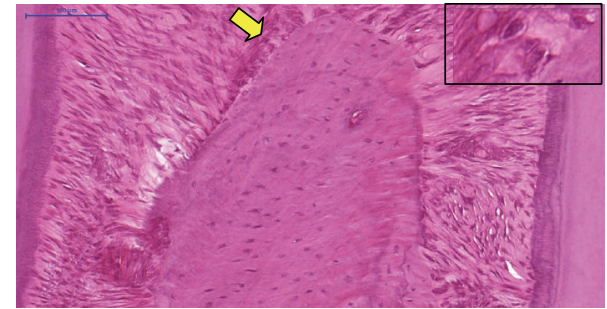

(b)

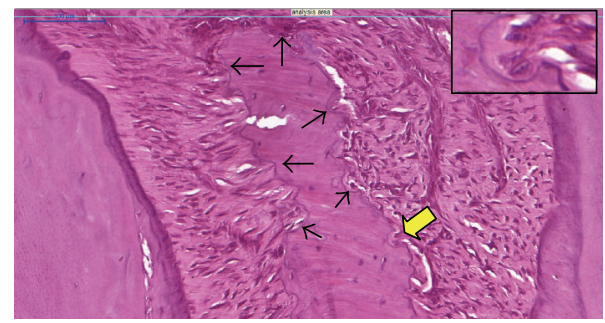

(d)

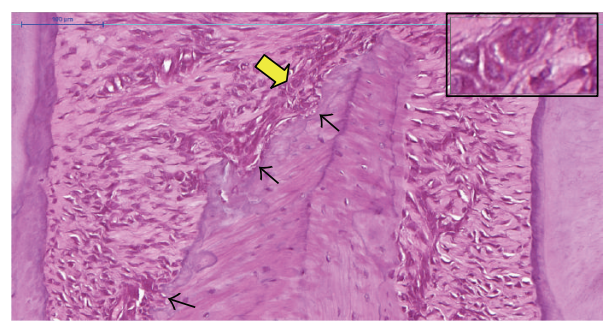

(e)

FIGURE 9: H\&E of osteoclast activity in baseline rats, healthy rats, and periodontitis rats (at 5x magnification). AB: alveolar bone, ABC: alveolar bone crest, C: cementum, D: dentine, and PDL: periodontal ligament. Osteoclast cell represented with yellow arrow and zone of bone destruction represented by black arrow. (a) shows no osteoclast activity in the baseline rat at zero day. (b) shows few osteoclasts in nonligated rats fed with saline (NLS) at 15 days. (c) shows few osteoclasts in nonligated rats fed with Gelam honey (NLH) at 15 days. (d) shows increased osteoclast activity in the ligated rats fed with saline $3 \mathrm{~g} / \mathrm{kg}$ at 15 days. (e) Shows increased osteoclast activity in the ligated rats fed with Gelam honey $3 \mathrm{~g} / \mathrm{kg}$ at 15 days.

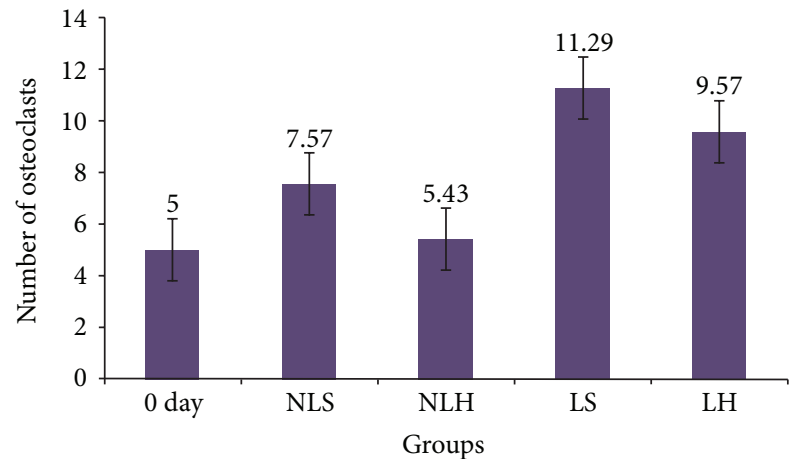

FIGURE 10: Mean number of osteoclasts among groups. NLS: nonligated saline, NLH: nonligated honey, LS: ligated saline, and LH: ligated honey.

alveolar bone loss. Their team concluded that the lack of effect on bone resorption may be related to a time delay to reach levels high enough for biological effects of curcumin on alveolar bone. Hence, this may clarify the inability of Gelam

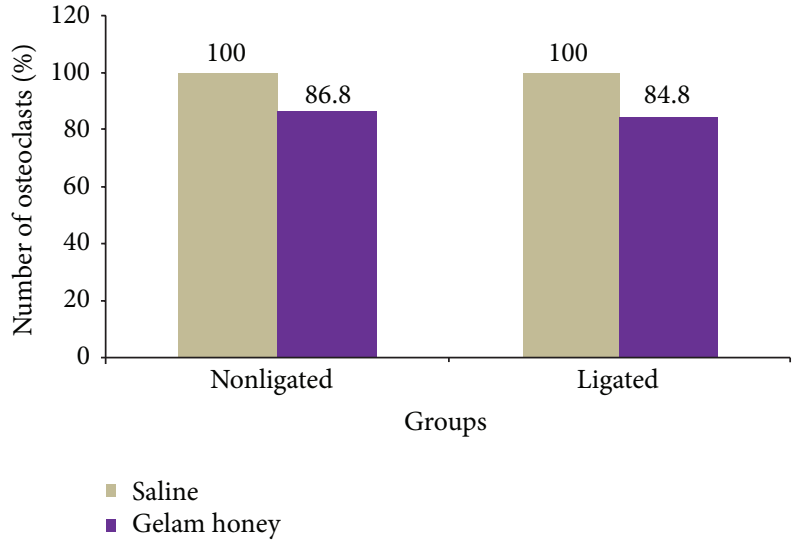

FIGURE 11: Percentage (\%) number of osteoclasts in nonligated and ligated groups.

honey to show effect on alveolar bone resorption evaluated radiographically in our study.

On the contrary, Toker et al. [7] reported that propolis was able to prevent alveolar bone loss in rat model when 
administered systemically for 11 days. We suggest that it might be due to different components in the honey and propolis especially that the flavonoids in propolis are higher 5-26\% [31] while in pure honey flavonoids content is only $0.006 \%$ [32]. Therefore, honey might take a longer duration to show an effect on alveolar bone compared to propolis. However, according to Kuhr et al. [10], episodes of alveolar bone loss peak until day 15 and a stagnant period of alveolar bone loss up to 60 days were observed. Thus, extending the duration of Gelam honey supplementation in our study might prevent further alveolar bone loss.

Many other types of honey or their extracts have been proven to be useful as an adjunct in treating periodontitis and have been commercialized. English et al. [33] reported that chewing gum containing Manuka honey reduced plaque index by $35 \%$. Propolis was proven to have potential used as a natural mouthwash, as an alternative to chemical mouthwashes [34]; Persian Thymus vulgaris honey was used as topical application in promoting wound healing after periodontal surgery [35].

In conclusion, though Gelam honey did not inhibit alveolar bone loss, it shows ability to reduce number of osteoclasts in induced periodontal disease among Sprague-Dawley rats when analysed using the histomorphometric method. Based on findings from this study and other previous studies which have proven the antibacterial, anti-inflammatory, antioxidant, and antimicrobial effects of Gelam honey, we highly recommended further study using Gelam honey or its extracts for treating periodontal disease. Therefore, this research may be useful for development of Gelam honey as a local therapeutic agent replacing drugs and chemical agents in the market currently.

\section{Conflict of Interests}

The authors declare that there is no conflict of interests regarding the publication of this paper.

\section{Acknowledgments}

This research was financially supported by the institution (University Kebangsaan Malaysia) under the "Dana Rangsangan Geran Universiti Penyelidikan, GUP Khas-UKM-GUP2011-091." The authors also thank all personnel who were directly or indirectly involved in this research study.

\section{References}

[1] R. Assuma, T. Oates, D. Cochran, S. Amar, and D. T. Graves, "IL-1 and TNF antagonists inhibit the inflammatory response and bone loss in experimental periodontitis," The Journal of Immunology, vol. 160, no. 1, pp. 403-409, 1998.

[2] G. M. P. Galbraith, C. Hagan, R. B. Steed, J. J. Sanders, and T. Javed, "Cytokine production by oral and peripheral blood neutrophils in adult periodontitis," Journal of Periodontology, vol. 68, no. 9, pp. 832-838, 1997.

[3] G. N. Michael, T. Henry, R. K. Perry, and A. Fermin, Carranza's Clinical Periodontology, Elsevier Health Sciences, 11th edition, 2012.
[4] R. Di Paola, S. Marzocco, E. Mazzon et al., "Effect of aminoguanidine in ligature-induced periodontitis in rats," Journal of Dental Research, vol. 83, no. 4, pp. 343-348, 2004.

[5] R. L. Webber, U. E. Ruttimann, and R. A. Groenhuis, "Computer correction of projective distortions in dental radiographs," Journal of Dental Research, vol. 63, no. 8, pp. 1032-1036, 1984.

[6] C. H. Li and S. Amar, "Morphometric, histomorphometric, and microcomputed tomographic analysis of periodontal inflammatory lesions in a murine model," Journal of Periodontology, vol. 78, no. 6, pp. 1120-1128, 2007.

[7] H. Toker, F. Ozan, H. Ozer, H. Ozdemir, K. Eren, and H. Yeler, "A morphometric and histopathologic evaluation of the effects of propolis on alveolar bone loss in experimental periodontitis in rats," Journal of Periodontology, vol. 79, no. 6, pp. 1089-1094, 2008.

[8] A. L. Dumitrescu, S. Abd El-Aleem, B. Morales-Aza, and L. F. Donaldson, "A model of periodontitis in the rat: effect of lipopolysaccharide on bone resorption, osteoclast activity, and local peptidergic innervation," Journal of Clinical Periodontology, vol. 31, no. 8, pp. 596-603, 2004.

[9] P. J. Baker, R. T. Evans, and D. C. Roopenian, "Oral infection with Porphyromonas gingivalis and induced alveolar bone loss in immunocompetent and severe combined immunodeficient mice," Archives of Oral Biology, vol. 39, no. 12, pp. 1035-1040, 1994.

[10] A. Kuhr, A. Popa-Wagner, H. Schmoll, C. Schwahn, and T. Kocher, "Observations on experimental marginal periodontitis in rats," Journal of Periodontal Research, vol. 39, no. 2, pp. 101106, 2004.

[11] V. de Lima, M. M. Bezerra, V. B. de Menezes Alencar et al., "Effects of chlorpromazine on alveolar bone loss in experimental periodontal disease in rats," European Journal of Oral Sciences, vol. 108, no. 2, pp. 123-129, 2000.

[12] E. Buduneli, S. Vardar, N. Buduneli et al., "Effects of combined systemic administration of low-dose doxycycline and alendronate on endotoxin-induced periodontitis in rats," Journal of Periodontology, vol. 75, no. 11, pp. 1516-1523, 2004.

[13] U. van der Velden, D. Kuzmanova, and I. L. C. Chapple, "Micronutritional approaches to periodontal therapy," Journal of Clinical Periodontology, vol. 38, no. 11, pp. 142-158, 2011.

[14] S. G. Ciancio, "Systemic medications: clinical significance in periodontics," Journal of Clinical Periodontology, vol. 29, no. 2, pp. 17-21, 2002.

[15] J. Slots and M. G. Jorgensen, "Effective, safe, practical and affordable periodontal antimicrobial therapy: where are we going, and are we there yet?" Periodontology 2000, vol. 28, no. 1, pp. 298-312, 2002.

[16] N. P. Lang and M. C. Brecx, "Chlorhexidine digluconate-an agent for chemical plaque control and prevention of gingival inflammation," Journal of Periodontal Research, vol. 21, no. 16, pp. 74-89, 1986.

[17] P. A. Nayak, U. A. Nayak, and R. Mythili, "Effect of Manuka honey, chlorhexidine gluconate and xylitol on the clinical levels of dental plaque," Contemporary Clinical Dentistry, vol. 1, no. 4, pp. 214-217, 2010.

[18] G. Bhat, P. Kudva, and V. Dodwad, "Aloe vera: nature's soothing healer to periodontal disease," Journal of Indian Society of Periodontology, vol. 15, no. 3, pp. 205-209, 2011.

[19] S. Z. Hussein, K. M. Yusoff, S. Makpol, and Y. A. M. Yusof, "Antioxidant capacities and total phenolic contents increase with gamma irradiation in two types of Malaysian honey," Molecules, vol. 16, no. 8, pp. 6378-6395, 2011. 
[20] A. M. Aljadi and K. M. Yusoff, "Isolation and identification of phenolic acids in Malaysian honey with antibacterial properties," Turkish Journal of Medical Sciences, vol. 33, no. 4, pp. 229236, 2003.

[21] R. Mohd Zohdi, Z. Abu Bakar Zakaria, N. Yusof, N. Mohamed Mustapha, and M. N. H. Abdullah, "Gelam (Melaleuca spp.) honey-based hydrogel as burn wound dressing," Evidence-Based Complementary and Alternative Medicine, vol. 2012, Article ID 843025, 7 pages, 2012.

[22] S. Z. Hussein, K. Mohd Yusoff, S. Makpol, and Y. A. Mohd Yusof, "Gelam honey inhibits the production of proinflammatory, mediators NO, $\mathrm{PGE}_{2}$, TNF- $\alpha$, and IL- 6 in carrageenan-induced acute paw edema in rats," Evidence-Based Complementary and Alternative Medicine, vol. 2012, Article ID 109636, 13 pages, 2012.

[23] M. Kassim, M. Achoui, M. Mansor, and K. M. Yusoff, "The inhibitory effects of Gelam honey and its extracts on nitric oxide and prostaglandin $\mathrm{E}_{2}$ in inflammatory tissues," Fitoterapia, vol. 81, no. 8, pp. 1196-1201, 2010.

[24] D. N. Liberman, R. M. Pilau, E. J. Gaio, L. F. Orlandini, and C. K. Rösing, "Low concentration alcohol intake may inhibit spontaneous alveolar bone loss in Wistar rats," Archives of Oral Biology, vol. 56, no. 2, pp. 109-113, 2011.

[25] M. R. Guimarães, L. S. Coimbra, S. G. de Aquino, L. C. Spolidorio, K. L. Kirkwood, and C. Rossa, "Potent anti-inflammatory effects of systemically administered curcumin modulate periodontal disease in vivo," Journal of Periodontal Research, vol. 46, no. 2, pp. 269-279, 2011.

[26] G. N. Michael, T. Henry, R. K. Perry, and A. Fermin, Carranza's Clinical Periodontology, Elsevier Health Sciences, New York, NY, USA, 11th edition, 2012.

[27] Y. Samejima, S. Ebisu, and H. Okada, "Effect of infection with Eikenella corrodens on the progression of ligature-induced periodontitis in rats," Journal of Periodontal Research, vol. 25, no. 5, pp. 308-315, 1990.

[28] X. Cai, C. Li, G. Du, and Z. Cao, "Protective effects of baicalin on ligature-induced periodontitis in rats," Journal of Periodontal Research, vol. 43, no. 1, pp. 14-21, 2008.

[29] K. H. Kwon, A. Murakami, and H. Ohigashi, "Suppressive effects of natural and synthetic agents on dextran sulfate sodium-induced interleukin- $1 \beta$ release from murine peritoneal macrophages," Bioscience, Biotechnology and Biochemistry, vol. 68, no. 2, pp. 436-439, 2004.

[30] N. Matsuo, K. Yamada, K. Shoji, M. Mori, and M. Sugano, "Effect of tea polyphenols on histamine release from rat basophilic leukemia (RBL-2H3) cells: the structure-inhibitory activity relationship," Allergy, vol. 52, no. 1, pp. 58-64, 1997.

[31] I. Kosalec, M. Bakmaz, S. Pepeljnjak, and S. Vladimir-Knežević, "Quantitative analysis of the flavonoids in raw propolis from northern Croatia," Acta Pharmaceutica, vol. 54, no. 1, pp. 65-72, 2004.

[32] M. De Graca Ribeiro Campos, S. Sabatier, M.-J. Amiot, and S. Aubert, "Characterization of flavonoids in three hive products: bee pollen, propolis, and honey," Planta Medica, vol. 56, no. 6, pp. 580-581, 1990.

[33] H. K. P. English, A. R. C. Pack, and P. C. Molan, "The effects of manuka honey on plaque and gingivitis: a pilot study.," Journal of the International Academy of Periodontology, vol. 6, no. 2, pp. 63-67, 2004.

[34] V. Dodwad and B. Kukreja, "Propolis mouthwash: a new beginning," Journal of Indian Society of Periodontology, vol. 15, no. 2, pp. 121-125, 2011.
[35] M. K. Samani, A. P. Bejeh, G. Mir, B. Sajadi, and S. M. Fereshtehnejad, "The potential of honey to promote wound healing in periodontology: a pilot randomized clinical trial," Medical Journal of the Islamic Republic of Iran, vol. 25, no. 4, pp. 177-185, 2011. 


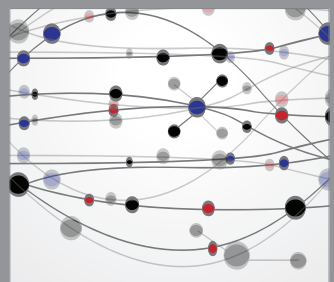

The Scientific World Journal
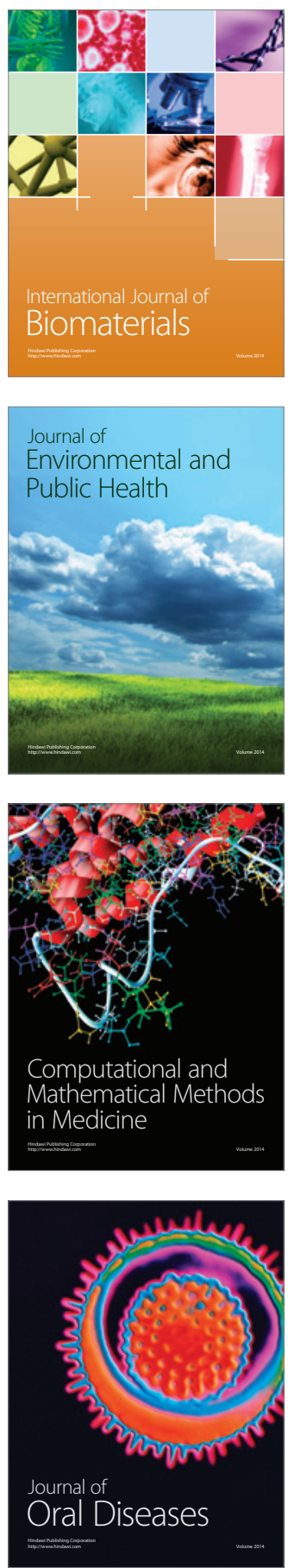
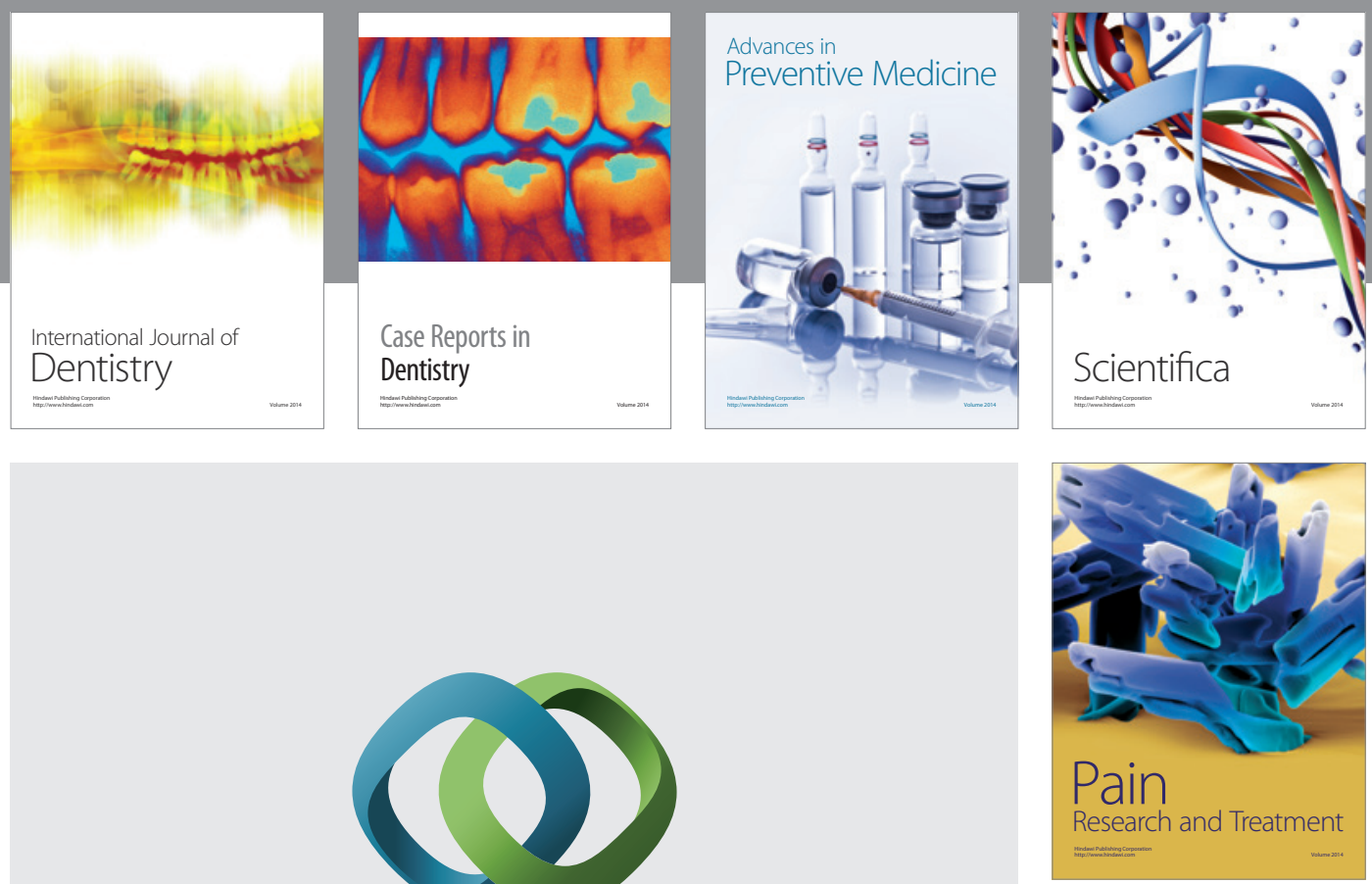

\section{Hindawi}

Submit your manuscripts at

http://www.hindawi.com
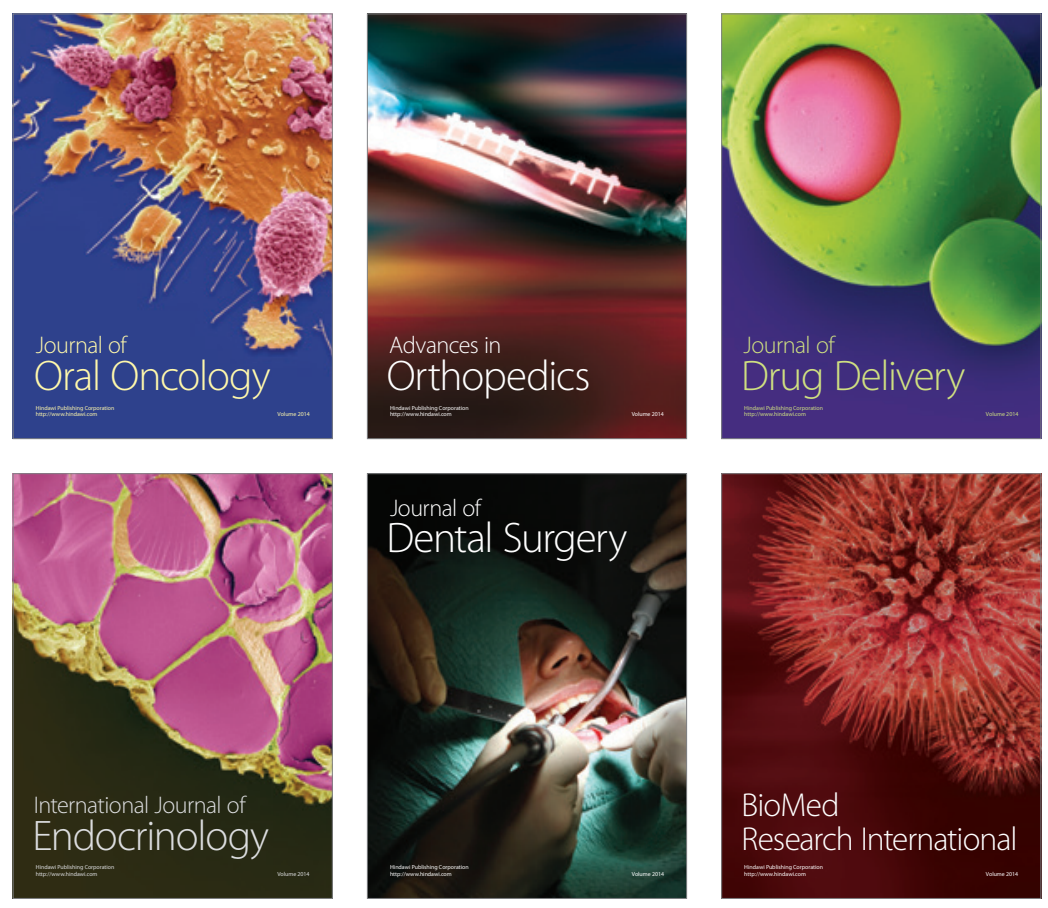

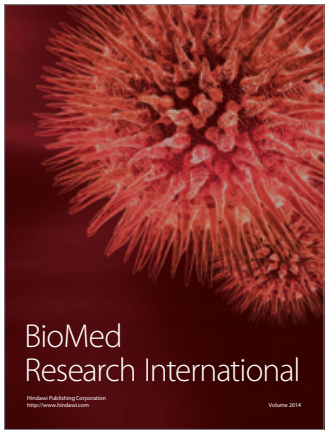

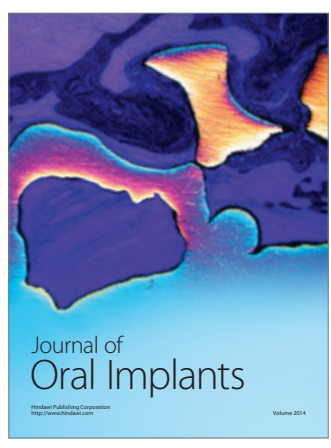
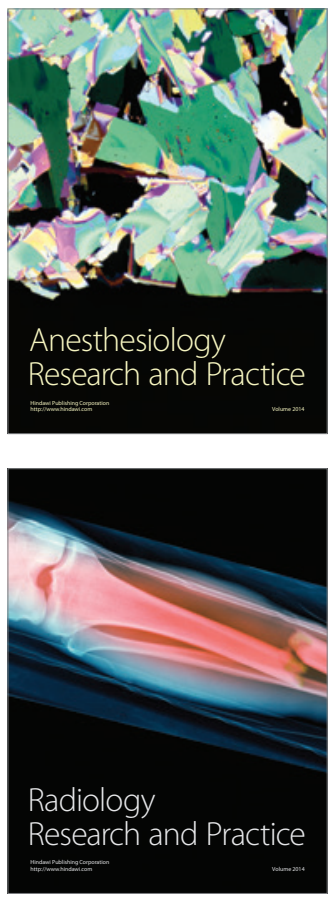